\title{
The role of the organic shell in hybrid molecular materials for EUV lithography
}

\author{
Lianjia Wu, ${ }^{a}$ Michaela Vockenhuber, ${ }^{b}$ Yasin Ekinci, ${ }^{b}$ Sonia Castellanos $^{a} *$ \\ ${ }^{a}$ Advanced Research Center for Nanolithography, Science Park 106, Amsterdam 1098XG, The \\ Netherlands; ${ }^{b}$ Paul Scherrer Institute, Forschungstrasse 111, Villigen 5232, Switzerland.
}

\begin{abstract}
As EUV lithography is being introduced into the market, the need for resists that can satisfy the exigent requirements of sensitivity, resolution, and line-edge roughness requires the development of guidelines for new generations of materials. In this work, we aim for gaining more understanding on the contribution of the organic components in inorganic-organic hybrid molecular resists to their sensitivity. A method to investigate the effect of specific functionalities on the sensitivity of metal oxo clusters is presented, which consists in the "doping" of the methacrylate shell through the introduction of fluorinated, iodinated and extended aromatic ligands. By introducing each of these functionalities in small ratios to the methacrylate ligand (1:11 and 2:10) the main chemical properties of the materials (solubility and molecular packing in the thin film) are expected to be unaltered. Our investigations show that the sensitivity is enhanced in the presence of halogens and drastically decreased when extended aromatic compounds are introduced. The trends are uncorrelated with the absorptivity estimated for each material, which seems to indicate that the doping functionalities might interfere with the cross-linking of the methacrylate ligands or introduce new chemical pathways. Our results indicate that specific organic functionalities can be used to tune the reactivity of hybrid compounds for EUV light by introducing them in doping amounts, thus preserving the processability of the initial material.
\end{abstract}

Keywords: molecular chemistry, spectroscopy, sensitivity, metal oxo cluster, doping

\section{INTRODUCTION}

The main motivation for the introduction of inorganic resists in EUV lithography was the need for higher EUV absorption cross-sections. While the enhancement of absorptivity is indeed a major advantage of these materials compared to polymer-based chemically amplified resists (CAR), there are many other chemical features in inorganic resists that can be advantageous if properly tuned. Inorganic resists are typically based on discrete entities (molecules or nanoparticles) that can act as small voxels in the lithographic progress, which is seen as favorable for the reduction of pattern roughness. ${ }^{1-3}$ They are also not relying on an amplification mechanism (or at least, not one based on photoacid generators), which simplifies the system and reduces stochastic sources. In addition, inorganic resists are expected to present less electron blur due to the different scattering of electrons when the inorganic units are present in the thin film compared to polymeric materials. ${ }^{4}$

In most cases, the inorganic component is coated and/or stabilized by an organic component. For many of these hybrid resists, the organic shell confers the solubility properties of the material, so that, once the latter is cleaved, this leads to the formation of less soluble, cross-linked inorganic networks. ${ }^{5-7}$ However, some works have evidenced that the organic shell can play a more active role in the patterning mechanism. ${ }^{2,8-11}$ The organic moieties can also promote the formation of new bonds, especially when they have terminal double bonds that are prone to undergo radical polymerization. This phenomenon was observed in molecular metallorganic resists (MORE) ${ }^{8}$ and in oxoclusters. ${ }^{9,10}$ Ober's group proposed that EUV irradiation can induce the replacement of ligands in $\mathrm{ZrO}_{2}$ and $\mathrm{HfO}_{2}$ nanoparticles by the sulphonic acid generated in the process, thus rendering the solubility switch. ${ }^{11}$ Recently, this concept was also applied to smaller molecules based on Zn-dimers where, as a result of EUV exposure, ligands with positive charge density at their terminal positions where replaced with by ligands with terminal negative charge density, leading to a solubility switch due to a change in the molecular polarity. ${ }^{2}$

Therefore, the design of new EUV photoresists materials that can meet all the requirements to keep up with Moore's law demands the understanding of the role of both inorganic and organic components in this new types of hybrid resist. With this in mind, we performed a study on the effect of small variations in the organic shell of a series of molecular hybrid

*s.castellanos@arcnl.nl 
compounds that shared a common inorganic core. The materials of choice are metal oxo clusters (MOCs). MOCs comprise a dense multimetallic inorganic core with a fixed number of metallic atoms and bridging oxygens/hydroxide groups and a specific number of organic ligands that are responsible for the shape and stability of the inorganic core. Each organic ligand is bonded to either one or two metallic cations through a carboxylate group. An intrinsic property of such bonding is its dynamic character, meaning that, in solution, the carboxylate ligands can "walk" around the cluster ${ }^{12}$ or that in the presence of competing chelating agents, the ligands can be exchanged by another species. ${ }^{13-15}$

In this work, we exploit the dynamic behavior of MOCs to generate a library of "doped" materials. Starting with a methacrylate-based MOC as pristine material, we introduce a ligand with a particular functionality, such as extended (hetero)aromatic, fluorinated or iodinated moiety, in small ratios (i.e. less than $17 \%$ ). We found that the sensitivity of resulting MOCs towards EUV light was much affected by the introduction of these "doping ligands". These findings underline the contribution of the organic components in the reactivity and lithographic performance of hybrid EUV photoresists.

\section{MATERIALS AND METHODS}

\subsection{Materials}

Zirconium oxo methacrylate $\left(\mathrm{ZrMc}_{12}\right)$, $\mathrm{Zr}$ propoxide, methacrylate acid, fluorinated, iodinated and one of the extended aromatic carboxylic acids, as well as all the solvents were purchased from Sigma-Aldrich and used as received.

\subsection{Ligand exchange reaction and thin film deposition}

Shell-doped MOCs are prepared via ligand exchange reactions. Precursor $\mathrm{ZrMc}_{12}(5 \mathrm{mmol})$ was added to a solution of fluorinated carboxylic acid $(5 \mathrm{mmol})$ and $\mathrm{CHCl}_{3}(900 \mu \mathrm{L})$. The suspension was sonicated for 5 minutes. To the reaction mixture of $\mathrm{ZrMc}_{11} \mathrm{~F}$ was added $100 \mu \mathrm{L}$ amount of propylene glycol methyl ether acetate (PGMEA). The reaction mixture was again sonicated for 2 minutes and filtered through a $0.2-\mu \mathrm{m}$ syringe filter to remove any particles. The filtrate was spin-coated onto silicon substrates at $2100 \mathrm{rpm}$ for $30 \mathrm{~s}$ and thin films were prebaked at $90{ }^{\circ} \mathrm{C}$ for $30 \mathrm{~s}$ to remove residual solvents.

Thin films of other shell-doped MOCs were prepared with similar method. The exact number of ligands in the doped organic shell was determined by atomic concentration from X-ray photoelectron spectroscopy (XPS).

\subsection{EUV exposure and characterization}

EUV exposure was performed with the EUV interference lithography tool at the XIL-II beamline of Swiss Light Source (SLS) at the Paul Scherrer Institute (PSI). Exposed photoresists were developed in $\mathrm{CHCl}_{3}$ for $30 \mathrm{~s}$ and dried under nitrogen without post-exposure bake. The thickness of resists at each dose was measured using a Bruker atomic force microscopy (AFM) with the ScanAsyst mode and that of unexposed sample was determined by making a scratch and measuring. Patterning images were recorded using scanning electron microscopy (SEM) (FEI Verios 460) with a voltage of $1 \mathrm{kV}$.

\section{RESULTS AND DISCUSSIONS}

\subsection{Effect of doping ligand on sensitivity}

The reason to work at a "doping" level was an attempt to avoid a significant alteration in the physical properties of the original material. Mainly, we wanted to keep the solubility properties of the non-doped precursor compound in order to be able to work with similar developers and make the study more comparable. Although a certain dipole moment could be introduced by breaking the symmetry of the original $\mathrm{ZrMc}_{12}$ cluster, we expected that the exchange of 1 or $2 \mathrm{X}$ ligands out of $12(8 \%$ and $17 \%)$ to yield $\mathrm{ZrMc}_{11} \mathrm{X}$ or $\mathrm{ZrMc}_{10} \mathrm{X}_{2}$, respectively would not dramatically change the intermolecular interactions. 

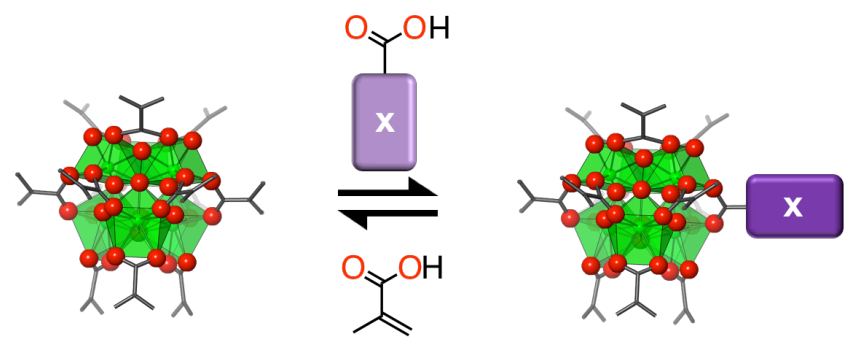

Figure 1. Schematic representation of the doping process of methacrylate-based MOC by ligand-exchange reaction containing a functionality $\mathrm{X}=$ fluorinated, iodinated, or extended aromatic system.

We therefore also considered safe to assume that the packing of the doped oxo clusters is quite close to that of the undoped material, given that the thin film presents a disordered arrangement of spherical molecules. This assumption allows us to estimate the densities of doped MOCs. The linear absorption coefficient $\alpha$ of each doped material was the calculated according to the equation $\alpha=\mu^{*} \delta / \mathrm{MW}$, where $\mu$ is the sum of atomic absorption cross-sections, $\delta$ is the density of the thin film and MW is the molecular weight. ${ }^{16}$ (Table 1).

Table 1. Calculated linear absorptivity of undoped $\mathrm{ZrMc}_{12}$ and doped $\mathrm{ZrMc}_{12-\mathrm{n}} \mathrm{X}_{\mathrm{n}}(\mathrm{n}=1$ or 2), where $\mathrm{X}$ is the doping ligand ( $\mathrm{I}=$ iodinated, $\mathrm{F}=$ fluorinated, Ar1/Ar2 = extended aromatic)

\begin{tabular}{|c|c|c|c|c|c|c|c|c|}
\hline MOC & $\mathrm{ZrMc}_{12}$ & $\mathrm{ZrMc}_{11} \mathrm{I}$ & $\mathrm{ZrMc}_{10} \mathrm{I}_{2}$ & $\mathrm{ZrMc}_{11} \mathrm{~F}$ & $\mathrm{ZrMc}_{10} \mathrm{~F}_{2}$ & $\mathrm{ZrMc}_{11} \mathrm{Ar} 1$ & $\mathrm{ZrMc}_{11}(\operatorname{Ar} 1)_{2}$ & $\mathrm{ZrMc}_{10}(\operatorname{Ar} 2)_{2}$ \\
\hline$\alpha\left(\mu m^{-1}\right)$ & 4.2 & 5.1 & 6.1 & 4.6 & 5.0 & 4.3 & 4.5 & 5.0 \\
\hline T@30 nm (\%) & 88 & 86 & 83 & 87 & 86 & 88 & 87 & 86 \\
\hline $\begin{array}{l}\text { absorbed } \\
\text { photons }\end{array}$ & - & $+22 \%$ & $+44 \%$ & $+10 \%$ & $+18 \%$ & $+3 \%$ & $+8 \%$ & $+18 \%$ \\
\hline
\end{tabular}

The choice of the doping ligands followed different criteria. In the case of the halogenated ligands, the main aim was to enhance the absorption and the yield of primary photoelectrons. Both fluorine and iodine have much higher EUV photon absorption cross-sections than carbon or zirconium. ${ }^{17}$ In addition, it had been reported that iodine can emit multiple electrons per absorbed photon. ${ }^{18}$ In the case of the extended aromatic fragments, two potential effects were anticipated. On the one hand, extended aromatic structures have lower band gaps than saturated carbon chains. It had been reported by Denbeaux and coauthors that a correlation had been found in chemically amplified resists between the band gap of the polymeric platform and their sensitivity. ${ }^{19}$ This was attributed to a likely higher secondary electron yield (more ionization events induced by the generated photoelectrons) occurring in polymers with lower bandgaps. On the other hand, we recently found that the introduction of extended aromatic structures in Ti-based oxo clusters provided them with the ability of stabilizing the radical cations that result from their photoionization. ${ }^{15}$ Therefore, with the introduction of extended aromatic structures in this work, our aim was to investigate which of these two phenomena would dominate in the sensitivity of the material. That is, an increase in secondary electrons due to a lower bandgap should lead to a sensitivity enhancement. Contrarily, stable radical cations could act as quenchers in the polymerization reaction involving the terminal bonds of the methacrylate ligands, the mechanism that had been found responsible for the solubility switch in methacrylate based Zr- and Hf-based metal oxo clusters.

Thin films of this series of doped materials were exposed to EUV in the XIL-II station at Paul Scherrer Institute. ${ }^{20}$ Using a multidose open frame exposure, the contrast curve for each compound was obtained. As in the case of the undoped precursor, all materials acted as negative tone resists and the same development procedure was used in all cases. 


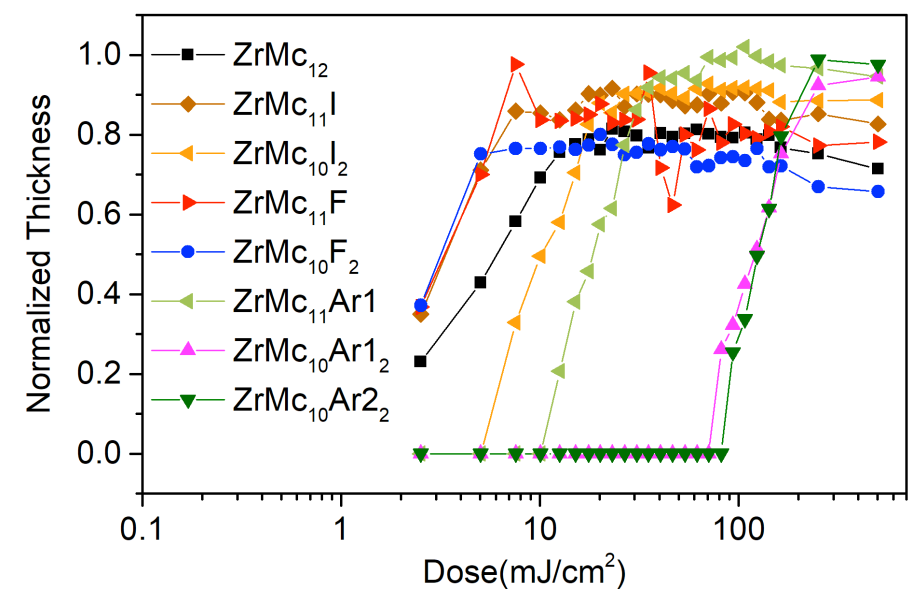

Figure 2. Contrast curves of doped Zr-based MOCs and $\mathrm{ZrMc}_{12-\mathrm{n}} \mathrm{X}_{\mathrm{n}}(\mathrm{n}=1$ or 2) obtained using the same development process $\left(\mathrm{CHCl}_{3}, 30 \mathrm{~s}\right)$.

It can be observed in Figure 2 that the presence of fluorine and iodine in the clusters has a positive effect in the sensitivity compared to the pristine material. This is in line with the slightly higher absorptivity of these materials. However, the trend does not follow a linear relationship with the photon absorption nor does the iodinated material show much lower doses to gel than the fluorinated material. In fact, an increase of the ratio of halogenated ligand did not seem to further increase the sensitivity. It should be noticed that in the case of the iodinated ligand, the incorporation of this particular ligand also means a decrease in the number of terminal double bonds in the organic shell. We suspect that this might be the reason for the negative effect in sensitivity in the case of the doubly doped $\mathrm{ZrMc}_{10} \mathrm{I}_{2}$. The influence on the sensitivity in the clusters doped with aromatic ligands is much more drastic. The dose to gel increases by one order of magnitude when a doping ratio of aromatic vs. methacrylate of 1:11 is used and up to 40x fold when the doping ratio is $2: 10$. The lack of proportionality between sensitivity and calculated absorptivity is thus even more evident for these compounds.

Therefore, we hypothesize that, although the absorptivity must influence, the changes in the sensitivity is strongly dependent on the contribution of new reaction pathways arising from the new ligands. The polarization of C-F and C-I bonds can favor the cleavage of these bonds. For instance, it is known that halogenated compounds can undergo dissociative electron attachment of the C-halogen bond. ${ }^{21,22}$ This new reactivity might further contribute to the solubility switch of the product, in addition to the cross-linking of the terminal double bonds in the methacrylate ligands. The drop of sensitivity for MOCs doped with extended aromatic ligands, on the other hand, could be related to the stabilization of the radical cations that are generated during the electron cascade. These species would act as hole traps, interrupting the radical or cationic polymerization of the terminal double bonds. They can also be termination points of those chain reactions, since these aromatic radical cations can uptake the electron or react with the propagating radical and stop the propagation process. Investigations to elucidate the exact mechanisms with spectroscopic methods are ongoing.

The contrast curves seem to further indicate an enhancement in the solubility contrast for all doped materials compared to the pristine one (Figure 3). This observation might be also related to the introduction of new reaction pathways parallel to (or competing with) the cross-linking reactions between methacrylate ligands, which could lead to new photoproducts that exhibited larger solubility contrast. Yet, further investigations involving solubility rates and the identification of the photoproducts are necessary to confirm and understand this effect. 


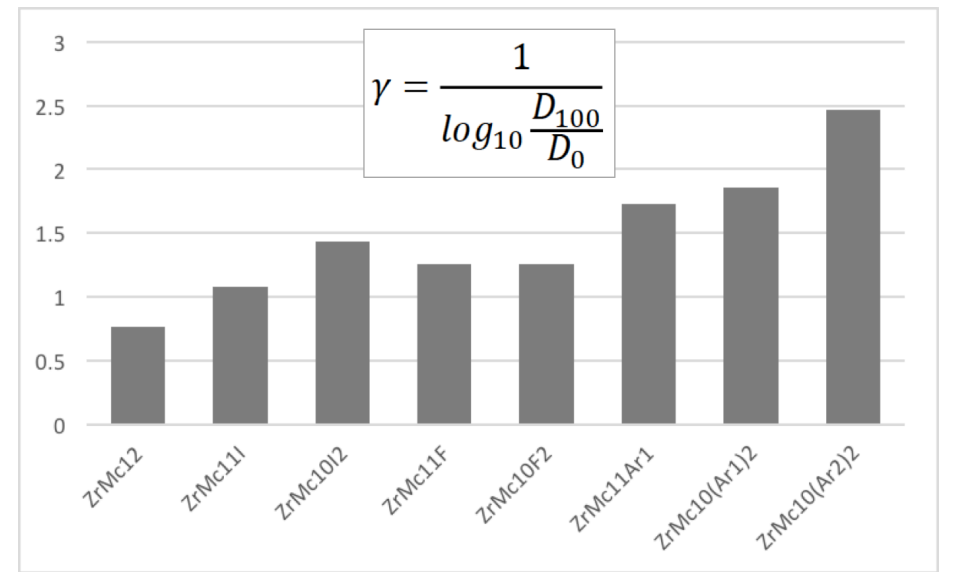

Figure 3. Calculated contrast for each doped Zr-MOC. $\mathrm{D}_{0}$ is dose-to-gel, which is the onset where the thickness of photoresist is measurable, estimated from linear fitting at the linear regime of the contrast curve. $D_{100}$ is the minimum dose at which the maximum thickness of converted material was found.

After observing the effect of the aromatic extended ligands on the sensitivity of the Zr-based materials, we performed the doping on the analogous $\mathrm{HfMc}_{12}$ MOC. The negative effect in the sensitivity was also reproduced on this compound. This result shows the potential of the ligand doping strategy on MOCs as EUV resists. Once the positive or negative effect of a particular ligand on the sensitivity is determined, the trend can be readily transferred to MOCs based on other metals.

\subsection{Effect of doping ligand on dense-lines and contact-hole/pillars printing}

Dense line patterning experiments by EUV interference lithography were performed on the fluorinated doped material $\mathrm{ZrMc}_{12-\mathrm{n}} \mathrm{F}_{\mathrm{n}}$. No optimization of the development process was carried out for this preliminary tests on the lithographic performance. Instead, the same developing conditions as for open frame experiments were used in order to compare with previous results obtained on the $\mathrm{ZrMc}_{12}$. Smaller pitches could be resolved for the doped material compared to the undoped $\mathrm{ZrMc}_{12}$ material (Figure 4). ${ }^{9}$
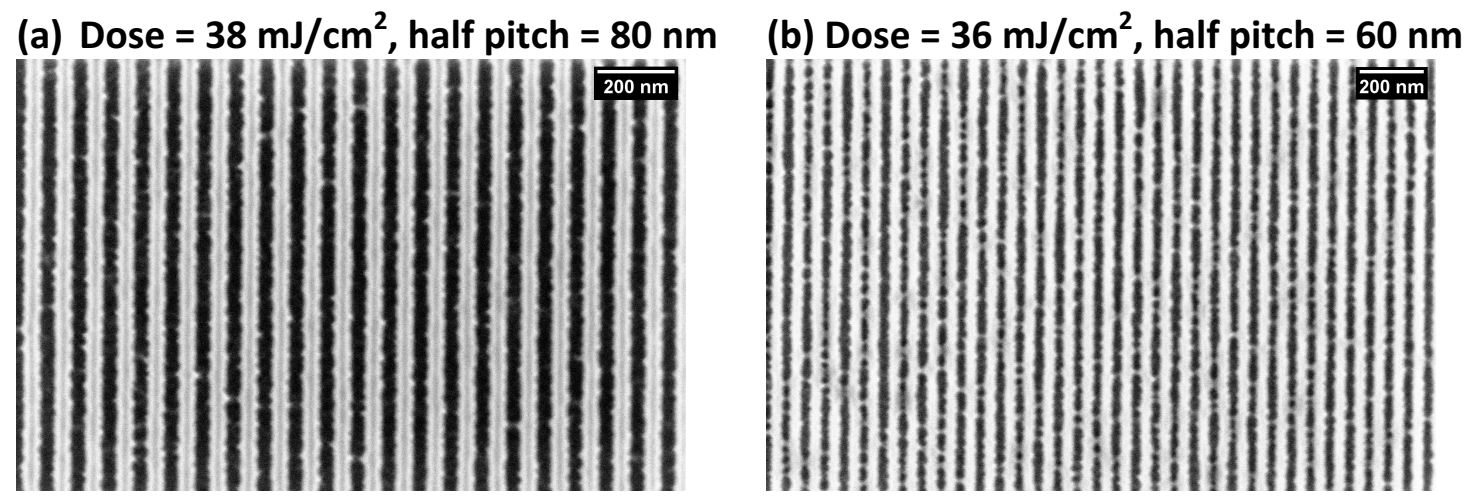

Figure 4. SEM images of dense line patterns on $\mathrm{ZrMc}_{11} \mathrm{~F}$.

The effect of the fluorinated doping ligand as well as of the extended aromatic ligand was also investigated for the printing of pillars. As for the dense line patterns, the development step was not optimized for these preliminary tests and chloroform was used as developer. The enhanced contrast of the doped materials compared to the pristine material seemed to enable the formation of this type of pattern. Pillars were resolved clearly for both the materials, although they presented scumming. In addition, the positive (for $\mathrm{ZrMc}_{11} \mathrm{~F}$ ) and negative (for $\mathrm{ZrMc}_{11} \mathrm{Ar} 2$ ) influence of the doping ligand on the sensitivity became evident in the dose to size of each material $\left(1.5 \mathrm{~mJ} / \mathrm{cm}^{2}\right.$ and $15 \mathrm{~mJ} / \mathrm{cm}^{2}$, respectively). The higher doses for $\mathrm{ZrMc}_{11} \mathrm{Ar} 2$ might account for the better quality for the pillar patterning. Mechanistic investigations and solubility studies for further optimisation of the lithographic performance of this type of materials are currently ongoing. 


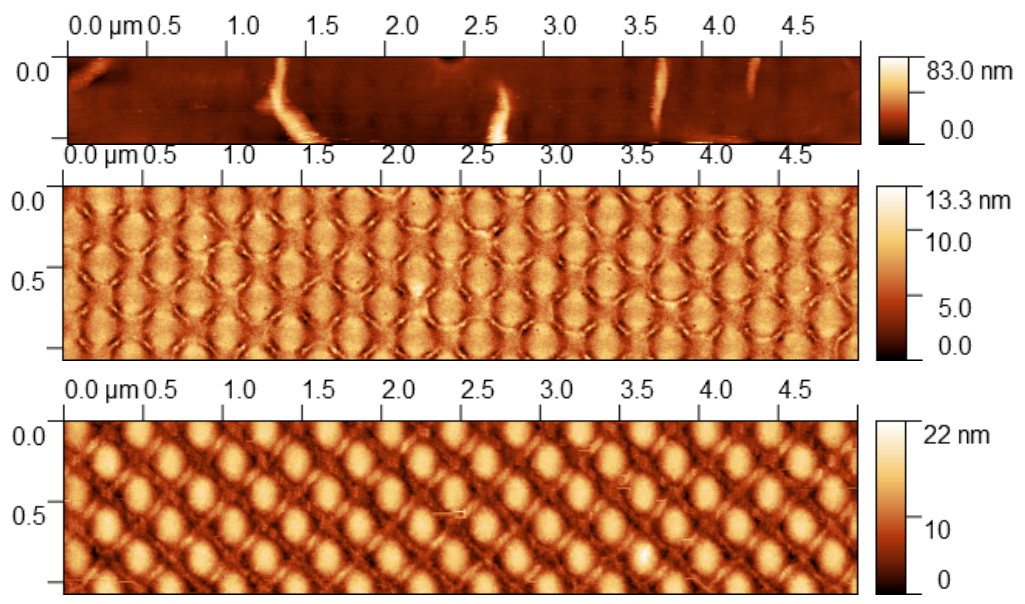

Figure 5. AFM images showing the doping effect in contact-holes/pillars. Top to botom: $\mathrm{ZrMc}_{12} 1.5 \mathrm{~mJ} / \mathrm{cm}^{2}, \mathrm{ZrMc}_{11} \mathrm{~F} 1.5$ $\mathrm{mJ} / \mathrm{cm}^{2}, \mathrm{ZrMc}_{11} \mathrm{Ar} 215 \mathrm{~mJ} / \mathrm{cm}^{2}$

\section{CONCLUSION}

The doping of the methacrylate shell in Zr-based metal oxoclusters (MOCs) with carboxylate ligands featuring fluorine, iodine or extended aromatic moieties has an important impact in the sensitivity of this hybrid molecular material that can perform as negative tone EUV resist. Ligands bearing fluorine or iodine had a positive effect in the sensitivity. Yet, this enhancement is not proportional to the estimated increase in absorptivity and thus might be the result of other (chemical) factors that add to photon absorption, such as electron induced bond dissociation reactions. Contrarily, the incorporation of extended aromatic structures has a detrimental effect in the sensitivity of the material, which is further stressed when more doping ligands are present in the cluster. We suspect that the ability of the extended aromatic structures to stabilize radical cations might interfere with the cross-linking of the methacrylate terminal double bonds, which is the mechanism that renders the solubility change in this type of resists. Although further investigations to reveal the mechanistic explanations of these effects are still ongoing, these results already highlight the great potential of MOCs as platforms to understand EUV chemistry and to identify which structural parameters can be used to tune lithographic performance. The synthetic versatility of MOCs, arising from the dynamic behavior of the carboxylate ligands, allow to easily attain a different combination of the two components (metals and ligands) of these hybrid resists. In this fashion, both the absorptivity (and likely the photoelectron yield) can be tuned by means of the metal content whereas the reactivity of the electron cascade can be modified by the organic shell.

\section{ACKNOWLEDGEMENTS}

We acknowledge the Paul Scherrer Institute, Villigen, Switzerland for the provision of beamtime at beamline XIL-II of the SLS (20180993). The research leading to these results has received funding from the European Community's Seventh Framework Programme (FP7/2007-2013) under grant agreement n. ${ }^{\circ} 312284$ (CALIPSO, 20180993).

\section{REFERENCES}

[1] A. Lio. "EUV resists: What's next?," Proc. SPIE 9776, 97760V (2016).

[2] H. Xu, K. Sakai, K. Kasahara, V. Kosma, K. Yang, H. C. Herbol, J. Odent, P. Clancy, E. P. Giannelis \& C. K. Ober. "MOF-inspired metal-containing clusters for high resolution patterning," Chem. Mater. 30, 4124-4133 (2018).

[3] L. Li, X. Liu, S. Pal, S. Wang, C. K. Ober \& E. P. Giannelis. "Extreme ultraviolet resist materials for sub-7 nm patterning," Chem. Soc. Rev. 2, 4855-4866 (2017).

[4] A. Vaglio Pret, T. Graves, D. Blankenship \& J. J. Biafore. "Modeling and simulation of low-energy electron scattering in organic and inorganic EUV photoresists," Proc. SPIE 10146, 1014609 (2017).

[5] W. D. Hinsberg \& S. Meyers. "A numeric model for the imaging mechanism of metal oxide EUV resists," Proc. 
SPIE 10146, 1014604 (2017).

[6] B. Cardineau, R. Del Re, H. Al-Mashat, M. Marnell, M. Vockenhuber, Y. Ekinci, C. Sarma, M. Neisser, D. A. Freedman \& R. L. Brainard. "EUV resists based on tin-oxo clusters," Proc. SPIE 90511, 90511B (2014).

[7] Y. Zhang, J. Haitjema, X. Liu, F. Johansson, A. Lindblad, S. Castellanos, N. Ottosson \& A. M. Brouwer. "Photochemical conversion of tin-oxo cage compounds studied using hard x-ray photoelectron spectroscopy," $J$. Micro/ Nanolithography, MEMS, MOEMS 16, 23510 (2017).

[8] R. Del Re, M. Sortland, J. Pasarelli, B. Cardineau, Y. Ekinci, M. Vockenhuber, M. Neisser, D. Freedman \& R. L. Brainard. "Low-LER tin carboxylate photoresists using EUV," Proc. SPIE 942221, 942221 (2015).

[9] S. Castellanos, L. Wu, M. Baljozovic, G. Portale, D. Kazazis, M. Vockenhuber, Y. Ekinci \& T. Jung. "Ti, Zr, and Hf-based molecular hybrid materials as EUV photoresists," Proc. SPIE 10583, 105830A (2018).

[10] E. C. Mattson, Y. Cabrera, S. M. Rupich, Y. Wang, K. A. Oyekan, T. J. Mustard, M. D. Halls, H. A. Bechtel, M. C. Martin \& Y. J. Chabal. "Chemical Modification Mechanisms in Hybrid Hafnium Oxo-methacrylate Nanocluster Photoresists for Extreme§ Ultraviolet Patterning," Chem. Mater. 30, 6192-6206 (2018).

[11] M. Kryask, M. Trikeriotis, C. Ouyang, S. Chakrabarty, E. P. Giannelis \& C. K. Ober. "Nanoparticle Photoresists: Ligand Exchange as a New, Sensitive EUV Patterning Mechanism," J. Photopolym. Sci. Technol. 26, 659-664 (2013).

[12] P. Walther, M. Puchberger, F. R. Kogler, K. Schwarz \& U. Schubert. "Ligand dynamics on the surface of zirconium oxo clusters," Phys. Chem. Chem. Phys. 11, 3640-3647 (2009).

[13] T. Frot, S. Cochet, G. Laurent, C. Sassoye, M. Popall, C. Sanchez \& L. Rozes. "Ti8O8(OOCR)16, a new family of titanium-oxo clusters: Potential NBUs for reticular chemistry," Eur. J. Inorg. Chem. 8, 5650-5659 (2010).

[14] J. Kreutzer, M. Puchberger, C. Artner \& U. Schubert. "Retention of the cluster core structure during ligand exchange reactions of carboxylato-substituted metal oxo clusters," Eur. J. Inorg. Chem. 2015, 2145-2151 (2015).

[15] L. Wu, M. Tiekink, A. Giuliani, L. Nahon \& S. Castellanos. "Tuning photoionization mechanisms of molecular hybrid materials for EUV lithography applications," J. Mater. Chem. C 7, 33 (2019).

[16] R. Fallica, J. Haitjema, L. Wu, S. Castellanos, A. M. Brouwer \& Y. Ekinci. "Absorption coefficient of metalcontaining photoresists in the extreme ultraviolet," J. Micro/ Nanolithography, MEMS, MOEMS 17, 23505 (2018).

[17] B. L. Henke, E. M. Gullikson \& J. C. Davis. "X-ray interactions: photoabsorption, scattering, transmission, and reflection at E=50-30000 eV, Z=1-92," At. Data Nucl. Data Tables 54, 181-342 (1993).

[18] D. F. Ogletree. "International Workshop on EUV Lithography 2017," in X-Rays, Electrons and Lithography: Fundamental Processes in Molecular Radiation Chemistry (2017).

[19] S. Grzeskowiak, J. Kaminsky, S. Gibbons, M. Murphy, J. Chandonait, R. L. Brainard \& G. Denbeaux. "Polymer effects on PAG acid yield in EUV resists," Proc. SPIE 10586, 105860D (2018).

[20] N. Mojarad, J. Gobrecht \& Y. Ekinci. "Interference lithography at EUV and soft X-ray wavelengths: Principles, methods, and applications," Microelectron. Eng. 143, 55-63 (2015).

[21] S. G. Rosenberg, M. Barclay \& D. H. Fairbrother. "Electron induced surface reactions of organometallic metal(hfac)2precursors and deposit purification," ACS Appl. Mater. Interfaces 6, 8590-8601 (2014).

[22] M. Mahmoodi-Darian, S. E. Huber, A. Mauracher, M. Probst, S. Denifl, P. Scheier \& T. D. Märk. "Temperature dependence of dissociative electron attachment to bromo-chlorotoluene isomers: Competition between detachment of $\mathrm{Cl}^{-}$and $\mathrm{Br}^{-}, "$ J. Chem. Phys. 148, 74301 (2018). 